\title{
Theoretical calculations of the primary defects induced by pions and protons in $\mathrm{SiC}$
}

\author{
S. Lazanu, I. Lazanu†, E. Borchi ${ }^{\ddagger}$ and M. Bruzzi ${ }^{\S}$
}

October 26, 2018

\begin{abstract}
In the present work, the bulk degradation of $\mathrm{SiC}$ in hadron (pion and proton) fields, in the energy range between $100 \mathrm{MeV}$ and $10 \mathrm{GeV}$, is characterised theoretically by means of the concentration of primary defects per unit fluence. The results are compared to the similar ones corresponding to diamond, silicon and GaAs.
\end{abstract}

Keywords:

SiC, Diamond, Silicon, Hadrons, Radiation damage properties

PACS:

61.80.Az: Theory and models of radiation effects.

61.82.-d: Radiation effects on specific materials.

\section{Introduction. General properties}

Silicon carbide is a new interesting material for use in optoelectronic and electronic devices, detectors, for the use in nuclear waste technology or in fusion reactors. In all these applications, this semiconductor material could be exposed to extreme conditions of temperature, to chemical agents and to radiation fields.

$\mathrm{SiC}$ is not a simple semiconductor, it is a whole class of semiconductors because of its polytypism [1]. Polytypism refers to one-dimensional polymorphism, i.e. the existence of different stackings of the basic structural elements along one direction. More than 200 polytypes have been reported [2]. Only few of them have practical importance. These include the cubic form $3 C(\beta)$, and the $4 H$ and $6 H$ hexagonal forms. For the cubic polytype, the symmetry group is $T_{d}^{2}$, while for the hexagonal ones this is $C_{6 v}^{4}$.

Some material characteristics of different polytypes of $\mathrm{SiC}$, useful for its utilisation in radiation fields, are presented in comparison with the corresponding ones of diamond, silicon and GaAs in Table 1. The compilation of data has been realised using Refs 3, 4, 5, 6, 6, 8, 9, 10, 11, 12.

The listed material properties could explain why electronic devices made from $\mathrm{SiC}$ can operate at extremely high temperatures without suffering from intrinsic conduction effects due to its wide bandgap. Also, this characteristic allows to emit short wavelength light when fabricated into a light emitting diode structure.

\footnotetext{
*National Institute for Materials Physics, POBox MG-7, Bucharest-Magurele, Romania

${ }^{\dagger}$ University of Bucharest, POBox MG-11, Bucharest-Magurele, Romania

$\ddagger$ Universita di Firenze, Dipartimento di Energetica, Via S. Marta 3, 50139 Firenze, Italy

$\S$ Universita di Firenze, Dipartimento di Energetica, Via S. Marta 3, 50139 Firenze, Italy
} 


\begin{tabular}{|c|c|c|c|c|c|c|}
\hline Material properties & Diamond & Silicon & $6 H \mathrm{SiC}$ & $4 H \mathrm{SiC}$ & $3 C \mathbf{S i C}$ & GaAs \\
\hline Crystal structure & Diamond [3] & Diamond [3] & Wurtzite [3] & Wurtzite [3] & Zincblende [3] & Zincblende [3] \\
\hline Crystal system & Cubic $[3]$ & Cubic [3] & Hexagonal [3] & Hexagonal [3] & Cubic $[3]$ & Cubic [3] \\
\hline Latice constant $[\mathrm{A}]$ & $3.567[3]$ & $5.431[3]$ & $\begin{array}{l}\text { a: } 3.08[3] \\
\text { c: } 15.12[3]\end{array}$ & $\begin{array}{l}\text { a: } 3.09[3] \\
\text { c: } 10.8[3]\end{array}$ & $4.38[3]$ & $5.85[3]$ \\
\hline Atomic number & 6 & 14 & $6 / 14$ & $6 / 14$ & $6 / 14$ & $31 / 33$ \\
\hline Atomic mass & 12.011 & 28.0855 & $12.01 / 28.08$ & $12.01 / 28.08$ & $12.01 / 28.08$ & $69.7 / 74.9$ \\
\hline Density $\left[\mathrm{g} / \mathrm{cm}^{3}\right]$ & $3.566[3]$ & $2.326[3]$ & $3.21[3]$ & & $3.17[3]$ & $5.32[3]$ \\
\hline Radiation length $\left[\mathrm{g} / \mathrm{cm}^{2}\right]$ & $42.7[4]$ & $21.82[4]$ & 28.9 & 28.9 & 28.9 & 12.2 \\
\hline Mobilities & e: $1800[5]$ & e:1450 [5] & e:450 [3] & e:900 [3] & e: $1000[3]$ & e: $8500[5]$ \\
\hline & $\mathrm{h}: 1200[5]$ & $\mathrm{h}: 450[5]$ & $\mathrm{h}: 50[3]$ & $\mathrm{h}: 100[3]$ & $\mathrm{h}: 100[3]$ & $\mathrm{h}: 400[5]$ \\
\hline Band gap $[\mathrm{eV}]$ & $5.47[5]$ & $1.12[5]$ & $3.02[3]$ & $3.26[3]$ & $2.2[3]$ & $1.43[5]$ \\
\hline Displacement energy & $43[9]$ & $21[10]$ & Si:35 & $\mathrm{Si}: 35[8]$ & Si: 35 & $10[10]$ \\
\hline averaged value $[\mathrm{eV}]$ & & & $\mathrm{C}: 20$ & $\mathrm{C}: 20[8]$ & $\mathrm{C}: 20$ & \\
\hline Dielectric constant & $5.5[6]$ & $11.8[6]$ & $9.61[6]$ & & $9.7[6]$ & $12.5[6]$ \\
\hline $\begin{array}{l}\text { Breakdown electric field } \\
\mathrm{V} / \mathrm{cm} \text { (for } 1000 \mathrm{~V} \text { operation) }\end{array}$ & & $2.5 \times 10^{5}$ & $2.4 \times 10^{5}$ & $2.2 \times 10^{5}$ & $2 \times 10^{6}$ & $3 \times 10^{5}$ \\
\hline $\begin{array}{l}\text { Thermal conductivity } \\
\text { @RT }\end{array}$ & $20[6]$ & $1.5[6]$ & $4.9[6]$ & $4.9[6]$ & $5.0[6]$ & $0.48[6]$ \\
\hline $\begin{array}{l}\text { Saturated electron drift velocity } \\
\mathrm{cms}^{-1} @ 2 \times 10^{5} \mathrm{Vcm}^{-1}\end{array}$ & $2.7 \times 10^{7}[6]$ & $10^{7}[6]$ & $2 \times 10^{7}[6]$ & $2.5 \times 10^{7}[6]$ & $10^{7}[6]$ & \\
\hline $\mathrm{dE} / \mathrm{dx}_{\min }\left[\mathrm{MeVcm}^{2} / \mathrm{g}\right]$ & $1.78[5]$ & $1.664[5]$ & 1.35 & 1.35 & 1.35 & $1.37[5]$ \\
\hline $\begin{array}{l}\text { Energy to form an electron- } \\
\text { hole pair }[\mathrm{eV}] @ \mathrm{RT}\end{array}$ & $13[7]$ & 3.72 & & $8.4[7]$ & & $4.6[7]$ \\
\hline
\end{tabular}
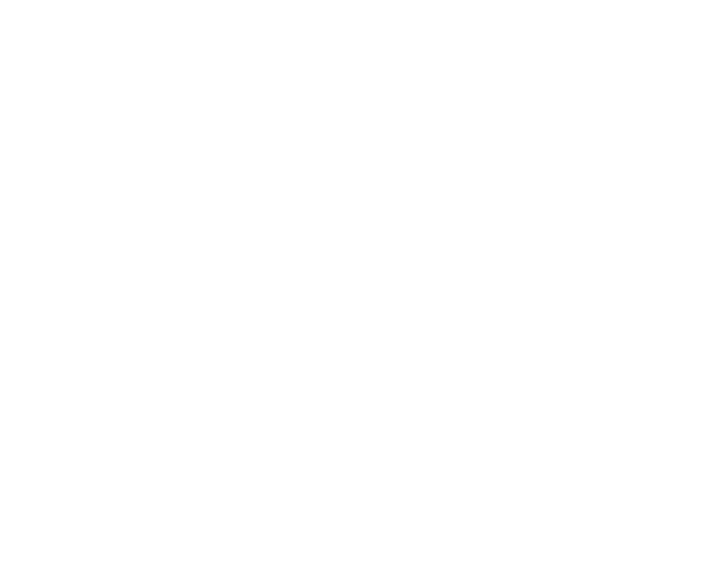
The high breakdown electric field, approximately one order of magnitude higher than the silicon or GaAs ones, and the high thermal conductivity, enable the fabrication of very high-voltage, high-power devices that dissipate large amounts of the excess heat generated. Additionally, it allows the devices to be placed very close together, providing high device packing density for integrated circuits. SiC devices can also operate at high frequencies (microwave) because of the high saturation electron drift velocity in the material.

Some SiC material characteristics are useful for detection applications: its radiation length lies between the corresponding values of diamond and silicon; its relatively wide band gap $(2.2 \div 3.3) \mathrm{eV}$ and a relative dielectric constant of 7.04 allows the assembly of detectors with low noise. With low mobilities: $450 \mathrm{~cm}^{2} \mathrm{~V}^{-1} \mathrm{~s}^{-1}$ for electrons and $115 \mathrm{~cm}^{2} \mathrm{~V}^{-1} \mathrm{~s}^{-1}$ for holes respectively, $\mathrm{SiC}$ does not permit a fast signal development and therefore is not able to operate in high fluxes of particles.

The crystalline materials for semiconductor devices used in high fluences of particles are strongly affected by the effects of radiation. Up to now, in spite of the experimental and theoretical efforts, most of the problems related to the behaviour of semiconductor materials in radiation fields, the identification of the induced defects and their characterisation, as well as the explanation of the degradation mechanisms are still open problems.

Diamond has the reputation of being a radiation hard material and is considered as a competitor to silicon. It is supposed that the concentration of primary defects induced in $\mathrm{SiC}$ in radiation fields is between the corresponding ones in diamond and silicon respectively.

\section{Mechanisms of degradation}

At the passage of the incident charged particle in the semiconductor material same of its energy is deposited into the target. The charged particles interact with both nuclei and electrons in a solid. The total rate of energy loss, could, in general, be divided artificially into two components, the nuclear and the electronic part.

The energy lost due to interactions with the electrons of the target gives rise to material ionisation, while the energy lost in interactions with nuclei is the origin of defect creation. The primary radiation defects are vacancies and interstitials.

There is not one physical quantity dedicated to the global characterisation of the effects of radiation in the semiconductor material. A possible one is the concentration of primary radiation induced defects per unit particle fluence $(C P D)$ 13. It is not proportional to the modifications of material parameters due to the irradiation because of the time evolution of vacancies and interstitials - see the discussion in reference 114]. Due to the fact that it does not exist a theory describing these processes of interaction, phenomenological models are used.

In this paper, the mechanism considered in the study of the interaction between the incoming particle and the solid, by which bulk defects are produced, is the following: the particle, heavier than the electron, with electrical charge or not, interacts with the electrons and with the nuclei of the crystalline lattice. The nuclear interaction produces bulk defects. As a result of the interaction, depending on the energy and on the nature of the incident particle, one or more light particles are produced, and usually one or more heavy recoil nuclei. These nuclei have charge and mass numbers lower or at least equal with those of the medium 15. After the interaction process, the recoil nucleus or nuclei, if they have sufficient energy, are displaced from the lattice positions into interstitials. Then, the primary knockon nucleus, if its energy is large enough, can produce the displacement of a new nucleus, and the process could continue as a cascade, until the energy of the nucleus becomes lower than the threshold for atomic displacements. Because of the regular 
nature of the crystalline lattice, the displacement energy is anisotropic. The primary interaction between the hadron and the nucleus of the lattice presents characteristics reflecting the peculiarities of the hadron, especially at relatively low energies. If the inelastic process is initiated by nucleons, the identity of the incoming projectile is lost, and the creation of secondary particles is associated with energy exchanges which are of the order of $\mathrm{MeV}$ or larger. For pion nucleus processes, the absorption, the process by which the pion disappears as a real particle, is also possible.

The energy dependence of cross sections, for proton and pion interaction with the nucleus, present very different behaviours: the proton-nucleus cross sections 16, 17] decrease with the increase of the projectile energy, have a minimum at relatively low energies, in the range of $100 \div 200 \mathrm{MeV}$, followed by a smooth increase, while the pion nucleus cross sections present for all processes a large maximum, at about $160 \mathrm{MeV}$, reflecting the resonant structure of interaction (the $\Delta_{33}$ resonance production), followed by other resonances, at higher energies, but with much less importance [18]. Because of the multitude of inelastic channels, in the concrete evaluation of defect production, the nuclear interactions has been modelled previously in some simplifying hypothesis, see refs. 13, 18, 19, 20.

The process of partitioning the energy of the recoil nuclei (produced due the interaction of the incident particle with the nucleus, placed in its lattice site) by new interaction processes, between electrons (ionisation) and atomic motion (displacements) is considered in the frame of the Lindhard theory [21].

\section{Results and discussions}

The concentration of the primary radiation induced defects per unit fluence in $\mathrm{SiC}$ has been calculated using the explicit formula:

$$
\begin{aligned}
C P D(E) & =\frac{N_{C}}{2 E_{d ; C}} \int \sum_{i}\left(\frac{d \sigma}{d \Omega}\right)_{i ; C} L\left(E_{R i}\right)_{S i C} d \Omega \\
& +\frac{N_{S i}}{2 E_{d ; S i}} \int \sum_{i}\left(\frac{d \sigma}{d \Omega}\right)_{i ; S i} L\left(E_{R i}\right)_{S i C} d \Omega \\
& =\frac{1}{N_{A}}\left[\frac{N_{C} A_{C}}{2 E_{d ; C}}(N I E L)_{S i C}^{C f a m i l y}+\frac{N_{C} A_{C}}{2 E_{d ; S i}}(N I E L)_{\text {SiC }}^{\text {Sifamily }}\right]
\end{aligned}
$$

where $E$ is the kinetic energy of the incident particle, $N_{C(S i)}$ is the atomic density of the $C(S i)$ in $\mathrm{SiC}, A_{C(S i)}$ is the atomic number of the $\mathrm{C}(\mathrm{Si}), E_{d ; C(S i)}$ - the threshold energy for displacements in the $\mathrm{C}(\mathrm{Si})$ sublattice of $\mathrm{SiC}, E_{R i}$ - the recoil energy of the residual nucleus produced in interaction $i, L\left(E_{R i}\right)$ - the Lindhard factor that describes the partition of the recoil energy between ionisation and displacements and $d \sigma_{i} / d \Omega$ - the differential cross section of the interaction between the incident particle and the nucleus of the lattice for the process or mechanism $i$, responsible in defect production. The atomic density $N_{C(S i)}$ in $\mathrm{SiC}$ is a material parameter which depends to the polytype through structural constants. The formula gives also the relation with the non ionising energy loss $(N I E L) . N_{A}$ is the Avogadro number. It is important to observe that there exists a proportionality between the $C P D$ and NIEL only for monoelement materials, and this proportionality can be extended only approximately for compounds with remote elements in the periodic table, e.g. for GaAs.

The $C P D$ allows the comparison of the effects produced by the same particle in different materials, while NIEL is especially used for the comparison of the effects produced in the same material by different particles. 
The partition of the energy of the incoming particle in the semiconductor material between ionisation and displacements has been calculated analytically, solving the general equations of the Lindhard theory in some physical approximations. Details about the hypothesis used could be found in reference [22]. In the particular case of the $\mathrm{SiC}$, a binary compound, the Lindhard curves have been calculated separately for each component, and the average weight Bragg additivity has been used. The energy spent into displacements is represented versus recoil energy for different recoils, characterised by their charge and mass numbers, in SiC, in Figure 1.

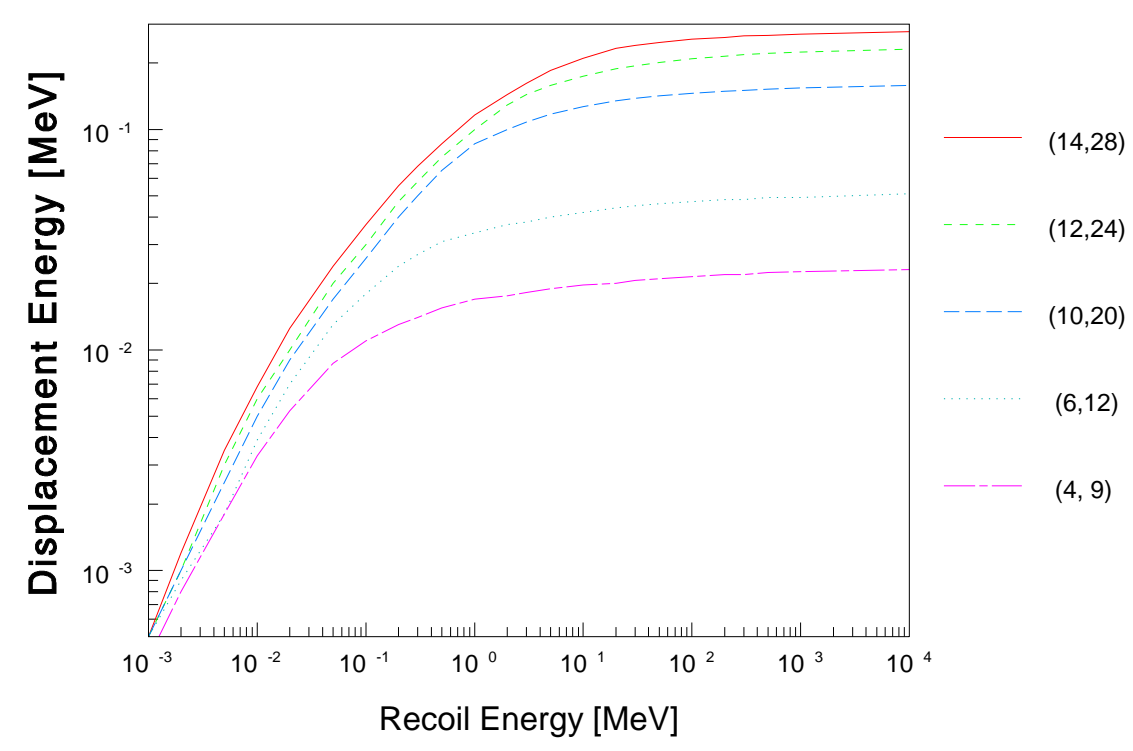

Figure 1: The displacement energy versus the energy of recoils (characterised by their atomic and mass number) in $\mathrm{SiC}$.

All curves start, at low energies, from the same curve; they have at low energies identical values of the energy spent into displacements, independent on the charge and mass number of the recoil. At higher energies, the curves start to detach from this main branch. This happens at lower energies if their charge and mass numbers are smaller. The maximum energy transferred into displacements corresponds to recoils of maximum possible charge and mass numbers (corresponding to the heaviest element, Si in this case). The curves present then a smooth increase with the energy. For the energy range considered here, the asymptotic limit of the displacement energy is not reached.

For the cross sections for proton and pion interactions with carbon and silicon, experimental data and theoretical extrapolations have been used - see references 19, 23, 24 and references cited therein. The cross sections for pion Ga and As nuclei are not available from experiment. Due to this fact, parametrisations of the energy dependence of the cross sections have been tried. Details about the procedure are given in reference 25.

As stated in Section 2, the minimum energy required to form a stable vacancy interstitial pair is usually called the threshold energy for displacements $\left(E_{d}\right)$. The 
regular nature of the crystalline lattice conduces to its anisotropy, atoms being more readily displaced along some directions than in others. This anisotropy is also the origin of differences in the rate production of defects as a function of orientation. In the literature, both calculated and experimental values for are reported for different crystals.

In principle, for $\mathrm{SiC}$, the situation is even more complicated and a threshold for atomic displacements could be defined for each polytype, for each atomic species, and for each direction in the crystal.

Some calculations have been reported in the literature for $E_{d}$ : in $\mathrm{SiC}$, along some directions of interest, by classical molecular-dynamics simulations using empirical potentials [26], and also using modern first-principle methods [27].

Experimental values are obtained from high voltage electron microscopy [28], energetic ion beam spectroscopy 29, and Rutherford backscattering analysis along different channelling directions 10,29, 30.

In the opinion of some authors [30], all these methods give consistent results, the recommended mean threshold energy for atomic displacements being of the order of $35 \mathrm{eV}$ on the $\mathrm{Si}$ sublattice, and of the order of $20 \mathrm{eV}$ on the $\mathrm{C}$ sublattice. These are the values we used in the calculations.

The energy dependence of the $C P D$ calculated (as stated above) for pions in $\mathrm{SiC}$, together with the estimated values for proton irradiation is presented in Figure 2 . In the same figure, for comparison, the $C P D$ produced by pions and protons in diamond [19, 24], silicon and GaAs are also represented. The values corresponding to protons in silicon are averages from the calculations reported in references 12$]$ and [31]. The curve for protons in GaAs is obtained from the results reported in reference [12] and is estimated by extrapolation in the energy range of interest for the present study, while the curve for pions in GaAs is from reference [23]. It could be observed that the degradations induced by pions and protons are very different and reflect the peculiarities of the interactions of the two particles with the nuclei of the semiconductor. For pions, the maximum of degradation is produced in the region of the $\Delta_{33}$ resonance, corresponding to about $140160 \mathrm{MeV}$ kinetic energy. In comparison with this behaviour, the maximum of the degradation is produced, for protons, at low energies, and a smooth and slow decrease of the $C P D$ is calculated with the increase of the energy. For the energy range considered in this paper, with the exception of the resonance region, the degradation induced by protons is higher than that for pions in $\mathrm{SiC}$, but for both incident particles, the concentration of primary defects produced per unit fluence are closed between 30 and $40 \mathrm{~cm}^{-1}$. The degradation induced by hadrons in $\mathrm{SiC}$ is between the diamond and silicon degradations.

Also, it could be observed that the pion degradation becomes more important with the increase of the mass number of the material, in the whole range of energy investigated.

The $C P D$ for $3 C, 4 H$ and $6 H$ polytypes of $\mathrm{SiC}$ has been evaluated separately, considering the structural characteristics of each polytype, and the same displacement energies for all of them; no significant differences between their behaviour in radiation fields has been found. These theoretical results are in agreement with the experimental data reported in reference [32].

\section{Summary}

In the present paper the concentration of primary induced defects per unit particle fluence has been calculated for the case of pion and proton irradiation, and the effects of these particles have been found intermediate in $\mathrm{SiC}$ between the corresponding ones in Si and diamond. 


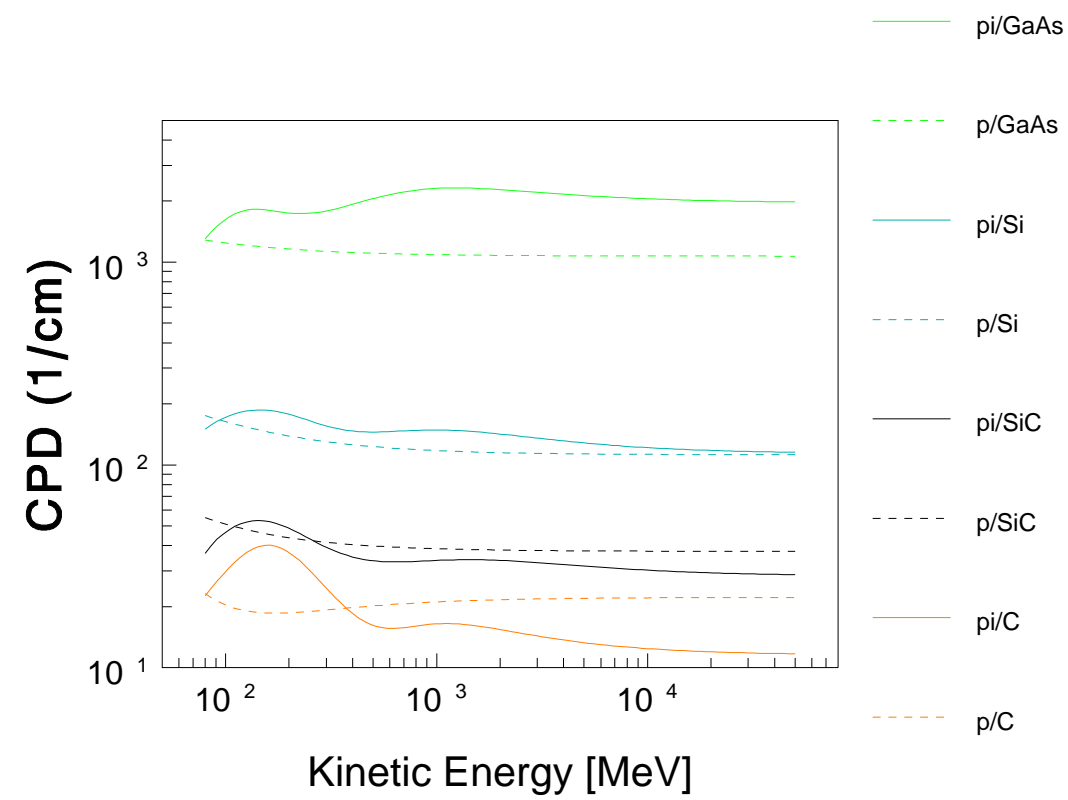

Figure 2: Concentration of primary induced defects on particle unit fluence for pions (continuous) and protons dashed) in (up to down): gallium arsenide, silicon, silicon carbide and diamond.

Negligible differences have been found between the $3 C, 4 H$ and $6 H$ polytypes in what regards the effects of pion and proton irradiation.

The Lindhard curves characterising the partition of the recoils energy between ionisation and displacements have been calculated using analytical approximations.

The main properties of $\mathrm{SiC}$ useful for its utilisation in radiation fields have been reviewed.

\section{References}

[1] A. P. Verma and P. Krishina, "Polymorphism and Polytypism in Crystals", Wiley, New York, 1966.

[2] W. R. L. Lambrecht, S. Limpijummong, S. N. Rashkeev and B. Segall, Phys. St. Sol. (b) 202, n.5 (1997) 5 .

[3] "Physical properties of WBG semiconductors", School of Chemical Engineering and Technology, Chenbuk National University, Koreea, http://che.chonbuk.ac.kr

[4] D. E. Groom et al., Review of Particle Physics, European Phys. J. 15 (2000).

[5] calculated

[6] G. Hall, Rept. Prog. Phys. 57 (1994) 481. 
[7] "Materials for High Temperature Semiconductor Devices", National Academy Press, 1995, http://books.nap.edu.

[8] "Physical and Electronic Properties of SiC", http://www.cree.com.

[9] M. Rogalla, K. Runge, A. Soldner-Rembold, Nucl. Phys. B (Proc. Suppl.) 78 (1999) 516.

[10] W.Jiang, S.Thevuthasan W.J.Weber, R.Grotzschel, Nucl. Instr. and Meth. in Phys. Res. B 161 (2000) 501.

[11] R. Kalise, in "The physics of diamond", Proc. Int. School of Physics Enrico Fermi, Italian Phys. Soc. 1997, eds. A. Paoletti and A. Tucchiarone.

[12] G. P. Summers, E. A. Burke, P. Shapiro, S. R. Messenger, R. J. Walters, IEEE Trans. Nucl. Sci., NS-40, (1990) 1372.

[13] I. Lazanu, S. Lazanu, E. Borchi, M. Bruzzi, Nucl. Instr. and Meth. Phys. Reseach, A 406 (1998) 259.

[14] I. Lazanu, S. Lazanu, U. Biggeri, E. Borchi, M. Bruzzi, Nucl. Instr. and Meth. Phys. Reseach, A 388 (1997) 370.

[15] H. H. K. Tang, IBM Journal of Research and Development, 40 (1996) 91.

[16] http://t2.lanl.gov.

[17] V. S. Barashenkov, preprint JINR Dubna P2-89-770, 1990.

[18] S. Lazanu, I. Lazanu, U. Biggeri, E. Borchi, M. Bruzzi, in Conf. Proc. Vol. 59, "Nuclear Data for Science and Technology", Eds. G. Reffo, A. Ventura and C. Gradi. (SIF, Bologna, 1997) p.1528.

[19] I. Lazanu, S. Lazanu, Nucl. Instr. and Meth. Phys. Reseach, A 432 (1999) 374.

[20] S. Lazanu, I. Lazanu, U. Biggeri, S. Sciortino, Nucl. Instr. and Meth. Phys. Reseach, A 413 (1998) 242.

[21] J. Lindhard, V. Nielsen, M. Scharff, P. V. Thomsen, Mat. Phys. Medd. Dan Vid. Sesk. 33 (1963) 1.

[22] S. Lazanu, I. Lazanu, Nucl. Instr. and Meth. in Phys. Res. A 462 (2001) 530.

[23] S.Lazanu, I.Lazanu, Nucl. Instr. and Meth. Phys. Reseach, A 419 (1998) 570.

[24] S.Lazanu, I.Lazanu, E.Borchi, Nucl. Phys. B (Proc. Suppl.) 78 (1999) 683.

[25] S. Lazanu, I. Lazanu, U. Biggeri, E. Borchi, M. Bruzzi, Nucl. Phys. B (Proc. Suppl.) 61 (1998) 409.

[26] R.Devanathan, W.J.Weber, T.Diaz de la Rubia, Nucl. Instr. and Meth. in Phys. Res. B 141 (1998) 118.

[27] W.Windl, T.J.Lenosky, J.D.Kress, A.F.Voter, Nucl. Instr. and Meth. in Phys. Res. B 141 (1998) 61.

[28] L.A.Honstvet, R.B.Smallman, P.M.Marquis, Phil. Mag. 41 (1980) 201.

[29] M.G.Grimaldi, L.Calgano, P.Musumoci, N.Frangis, J.Van Landuyt, J. Appl. Phys. 81 (1997) 7181. 
[30] W.Jiang, W.J.Weber, S.Thevuthasan, D.E.McCready, Nucl. Instr. and Meth. in Phys. Res. B 148 (1998) 557.

[31] A. van Ginneken, preprint Fermilab, FN-522 (1989).

[32] E. Wendler, A.Heft, W.Welsch, Nucl. Instr. and Meth. in Phys. Res. B 141 (1998) 10. 